\title{
1. Modelling the transport and adsorption dynamics of arsenic in a soil bed filter
}

Raka Mondal $^{\mathrm{a}}$, Sourav Mondal ${ }^{\mathrm{a}}$, Krishnasri V. Kurada ${ }^{\mathrm{b}}$, Saikat Bhattacharjee ${ }^{\mathrm{b}}$, Sourav Sengupta $^{\mathrm{b}}$, Mrinmoy Mondal ${ }^{b}$, Sankha Karmakar ${ }^{b}$, Sirshendu De ${ }^{\mathrm{b}}$, Ian M. Griffiths ${ }^{\mathrm{a}, *}$

${ }^{a}$ Mathematical Institute, University of Oxford, Oxford OX2 6GG, UK

${ }^{b}$ Department of Chemical Engineering, Indian Institute of Technology Kharagpur, Kharagpur 721302, India

\begin{abstract}
7 Abstract
Arsenic is among the most hazardous contaminants present in drinking water. Recent increase in agricultural growth and fertiliser use in India and Bangladesh has led to the release of naturally occurring arsenic from the rocks, creating a major public health issue. A novel technology has been developed using naturally abundant laterite soil to filter arsenic, providing potable water to more than 5000 people. To upscale this technology and realise its full potential, a comprehensive understanding of the dependence of filter life on operating regime (flow rate, arsenic concentration and filter size) is essential. We present a mathematical model that characterises arsenic removal, circumventing the need for time-consuming experiments. The model incorporates inter- and intra-particle mass transport within the filter medium. ${ }_{16}$ The resulting model enables prediction of a filter lifetime in a specified role, such as on a domestic or community scale, and should assist in future filter deployment and maintenance.

Keywords: arsenic removal; mathematical modelling; computational fluid dynamics; intra-particle dynamics; asymptotic analysis; India and Bangladesh
\end{abstract}

\section{Introduction}

The availability of safe drinking water is a necessity for sustainable life. Sources of drinking water, both underground and surface, are extremely susceptible to contamination, from physical, chemical, biological, or radioactive sources [1]. Currently, various water-purification technologies are globally adopted to remove contaminants. There are strict guidelines for safe drinking water, set by concerned agencies, such as the Environmental Protection Agency and the World Health Organization (WHO) that are closely monitored to ensure consumption of safe drinking water.

Arsenic $(A s)$, a naturally occurring element found in rocks and the earth's crust, is among the most hazardous contaminants in drinking water sources [2]. As is embedded in geological sources and enters into the water supply as the rocks are eroded by the flow of water from rivers and rainwater. Further, use of manure containing phosphate and other agricultural activities lead to the break down of arsenic binding bonds in different rocks, releasing As into the groundwater. Consequently, if not properly managed, the level of $A s$ contamination will continue to rise because of human activity and agricultural growth. At present, more than 200 million people and over 70 countries in the world are affected by $A s$ contamination [3]. A safe limit of $10 \mu \mathrm{g} / \mathrm{L}$ of $A s$ in drinking water was declared by WHO in 2000 [4, 5]. To address this issue effectively, it is necessary to have a water-purification technology that: (i) meets 
the safe drinking-water criteria; (ii) requires minimal energy; (iii) offers high throughput; (iv) is easy to scale up to cater for large population; and (v) generates minimal waste.

A novel strategy that achieves all five of these criteria has recently been discovered, using readily available laterite soil [6, 7]. Laterite is iron-rich and is able to remove $A s$ through adsorption. Raw laterite is treated chemically to enhance the surface area and increase adsorption capacity several fold.

However, as with any other adsorption medium, the filter has a certain lifespan, beyond which the filtrate no longer meets the safe limit for drinking water. For the design and successful implementation of this new filtration technology, it is therefore vital to predict the long-term behaviour of this technology. This long-term performance is influenced by the operating conditions, such as the input rate of the contaminated water, the mass of the adsorbent and the contaminant concentration level. Moreover, since the typical lifetime of such filters is of the order of several years, conducting experiments to determine the dependence of filter lifetime on the operating parameters is impractical. This therefore warrants the need of a suitable mathematical model to understand and characterise the operation and to predict the adsorption behaviour and filtration performance.

Several studies exist in the literature reporting on the theory of adsorption by different adsorbent media. The kinetics of $A s$ adsorption in water by raw laterite has been reported using a resistance-in-series model [7]. A shrinking-core model by Maiti et al. [8] was used to calculate the effective pore diffusivity and the external mass-transfer coefficient. Here, the adsorbent is assumed to be composed of spherical particles, around which the fluid flows and the contaminants are adsorbed. The adsorbed species penetrate into the adsorbent so that the core of unaffected material shrinks with time. A theory to explain the different steps involved in As removal from groundwater by adsorption has been reported by Maji et al. [9], determining the interplay of diffusion and adsorption. However, in these works, the kinetics of $A s$ adsorption was studied under well-stirred batch conditions. Such experiments do not simulate the actual removal performance when contaminated water flows past a stagnant bed of adsorbent particles. Thus, aforementioned models do not predict the long-term performance of an actual filter, in which hydrodynamics also plays a role. Semi-empirical and empirical models are available to quantify the performance of an adsorption bed under continuous operating conditions. However, these models are associated with certain assumptions, thereby limiting their applications. For example, the Adams-Bohart model assumes that the adsorption rate is proportional to the residual adsorption capacity and the contaminant concentration [10]. The Wolborska model assumes low fluid velocity through the column and dominance of diffusion over convection [11]. Again, however, none of these models account for the coupling of the fluid flow to the transport of the contaminants, despite its potentially significant role.

A key feature of $A s$ removal by laterite soil is the intra-particle dynamics. As is not only removed via surface adsorption onto laterite particles but through penetration into its core and adsorption within the internal pore structure. Employing a conventional adsorption-isotherm model for surface adsorption leads to an anomaly between the predicted and experimentally observed $A s$ removal rate.

In order to circumvent the limitations of semi-empirical and empirical models, in this paper we develop a theory based on first principles to describe the fluid flow through the laterite soil (a porous medium), which is coupled to the convective, diffusive and adsorptive transport for the contaminant. We also 
account for the transport dynamics within the porous adsorbent particle. The unknown $A s$ diffusivity in the porous medium as well as the intra-particle diffusion is determined by comparing with the results of simple experiments. The model is then fully specified and is used to predict two defining properties of the filter for any set of physical characteristics of the system, namely the lifetime and the breakthrough time of the filter. The breakthrough time is defined as the time when the adsorbent bed becomes exhausted with contaminant and loses the capacity for any further adsorption. The lifetime of the filter is the time when the concentration of contaminant in the exit stream reaches the safe limit for the intended purpose, which is reached before breakthrough. Beyond the lifetime, the filter is no longer fit to produce safe drinking water. Knowing the filter lifetime is imperative in the design of a filter unit and is an outcome of the model presented that cannot be estimated convincingly from simple semi-empirical models.

In this paper we will develop a theoretical model that predicts the operating lifetime of a filter given the input parameters, namely, the amount of treated laterite in the filter, the required flow rate, and the inlet $A s$ concentration. The model will be used to explore the adsorption behaviour within the particles, which cannot be assessed experimentally. The main objective of the model will be to use the short-term laboratory-scale experimental data to predict the long-term filtration behaviour and thus the lifetime of a field-scale filter. The model will also provide a predictive tool for upscaling, to determine the filter size required to generate a given flow rate for a given period to supply in a school or local community. At present, more than 20 filters have been deployed for domestic use and three filter assemblies have been established for community use. Predicting the precise lifetime of these filters under the real operating conditions remains a challenge [12]. We will use our model to extract a performance-lifetime relationship for field implementation providing a protocol for ensuring a safe and sustainable operation.

\section{Mathematical model}

A typical filter set-up consists of laterite soil housed in a cylindrical container into which the fluid enters uniformly across the top of the filter (see Fig. 1, left). We model the column using a cylindrical coordinate system $(r, \theta, z)$ with radial symmetry (Fig. 1, right).

The mathematical model comprises three different aspects: flow of the contaminated water through the interstitial space of the porous medium, and transport and adsorption of the contaminant species.

\subsection{Flow model}

The flow of incompressible fluid through the porous medium can be described using the continuity and Darcy-Brinkman equations [13]

$$
\begin{aligned}
\nabla \cdot \boldsymbol{u} & =0, \\
-\nabla p+\frac{\mu}{\phi} \nabla^{2} \boldsymbol{u}-\frac{\mu \boldsymbol{u}}{\kappa} & =0 .
\end{aligned}
$$

Here, $\boldsymbol{u}=u \boldsymbol{e}_{\boldsymbol{r}}+v \boldsymbol{e}_{\boldsymbol{z}}$ is the Darcy velocity, with $\boldsymbol{e}_{\boldsymbol{r}}$ and $\boldsymbol{e}_{\boldsymbol{z}}$ unit vectors in the $r$ and $z$ directions respectively, $\mu$ is the dynamic fluid viscosity, $p$ is the fluid pressure and $\kappa$ and $\phi$ are the permeability and porosity of the medium, respectively. The Darcy-Brinkman equation governs the flow in a general porous medium for arbitrary flow speeds, and a broad range of porosities and permeabilities, which may also 


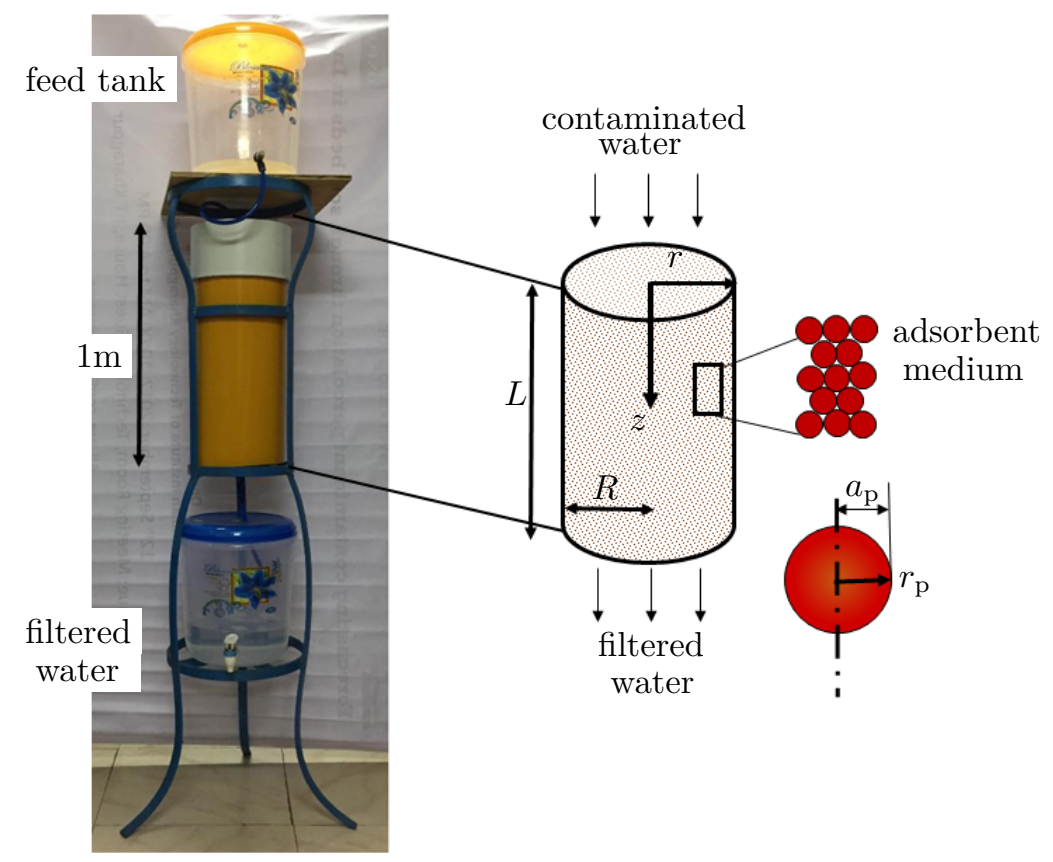

Figure 1: Left: A typical field-scale filter. Contaminated water is held in a feed tank that feeds into a column containing laterite adsorbent material. The filtered water seeps out of the bottom of the column. Right: Schematic of the filtration system. A cylinder of radius $R$ and height $L$ is packed with laterite adsorbent material. The adsorbent material is modelled as porous spheres of radius $a_{\mathrm{p}}$, which is unchanged by the adsorption of arsenic. We use coordinates $(r, z)$ to denote the position in the filter and $r_{\mathrm{p}}$ to denote the radial position in a particle. 
vary in space. Later, we will identify dimensionless parameters that determine the relative importance of the terms. In Eq. (2), the unknown variables $\boldsymbol{u}$ and $p$ are solved subject to the following initial and boundary conditions:

(i) At time $t=0$, we apply $u=v=0$ everywhere.

(ii) The inlet of contaminated water is distributed uniformly over the upper boundary, i.e., $u=0$ and $v=v_{\mathrm{i}}$, on $z=0$ and $0 \leq r \leq R$, where, $v_{\mathrm{i}}$ is a constant inlet velocity of contaminated water.

(iii) At the filter wall, $r=R$, and $0 \leq z \leq L$, we impose no-slip and no-penetration boundary conditions, i.e., $u=v=0$.

(iv) At the centre, $r=0$, we apply a symmetry condition, $u=\partial v / \partial r=0$.

(v) At the outlet, $z=L, 0 \leq r \leq R$, the device is open to the atmosphere, so $p=p_{a}$, which denotes atmospheric pressure.

In practice we expect the second-order term in (2) to be small. However, we choose to retain this here for generality. In addition, this term will be required to satisfy the no-slip conditions, (iii), that we impose on the walls of the filter. We therefore expect this term to become important near the boundaries of the filter device.

\subsection{Contaminant transport}

The transport of contaminant occurs on two distinct length scales, namely at the inter-particle and intra-particle level.

\subsubsection{Inter-particle transport}

The contaminant transport is modelled, for dilute concentrations, by the advection-diffusion equation

$$
\phi \frac{\partial c}{\partial t}+\boldsymbol{u} \cdot \nabla c=D_{\mathrm{l}} \nabla^{2} c-k_{\mathrm{f}} \xi\left(c-\left.c_{\mathrm{p}}\right|_{r_{\mathrm{p}}=a_{\mathrm{p}}}\right),
$$

where $c(r, z, t)$ is the contaminant $(A s)$ concentration $\left(\mathrm{kg} / \mathrm{m}^{3}\right), D_{\mathrm{l}}$ is the effective diffusion coefficient (constant) of contaminant in the porous bed, $k_{\mathrm{f}}$ is the mass-transfer coefficient at the particle interface, and $\left.c_{\mathrm{p}}\right|_{r_{\mathrm{p}}=a_{\mathrm{p}}}$ is the contaminant concentration at the particle surface ; $k_{\mathrm{f}}$ is less easily determined but may be related to other system properties as we will see later (Eq. (24)). We assume the particles to be spheres of radius $a_{\mathrm{p}}$ and $r_{\mathrm{p}} \in\left[0, a_{\mathrm{p}}\right]$ denotes the radial coordinate for the particle (see Fig. 1 and following section). The surface area factor exposed to the free fluid of the porous bed (not including inside the pores), $\xi$, for randomly packed spherical particles is [14]

$$
\xi=\frac{3}{a_{\mathrm{p}}}(1-\phi)
$$

Eq. (3) is subjected to the following initial and boundary conditions:

(i) Initially the filter does not contain any $A s$, and so $c=0$ everywhere at $t=0$.

(ii) At the inlet, $z=0$ and $0 \leq r \leq R$ the contaminant concentration is a constant, $c=c_{\mathrm{f}}$.

(iii) At the filter wall, $r=R$ and $0 \leq z \leq L$, we apply a no-flux condition, $\partial c / \partial r=0$.

(iv) At the centre, $r=0$, we apply a symmetry condition, $\partial c / \partial r=0$.

(v) At the outlet, $z=L$ and $0 \leq r \leq R$, we apply a zero-diffusive-flux condition, $\partial c / \partial z=0$, so that contaminant is purely advected out of the filter. This corresponds to a passive boundary condition, which does not require additional information about what happens once the water exits the filter. 
The continuity and Darcy-Brinkman equations, Eqs. (1) and (2) become

$$
\begin{aligned}
\bar{\nabla} \cdot \overline{\boldsymbol{u}} & =0, \\
-\bar{\nabla} \bar{p}+\frac{D a}{\phi} \bar{\nabla}^{2} \overline{\boldsymbol{u}}-\overline{\boldsymbol{u}} & =0,
\end{aligned}
$$

where $\bar{\nabla}=\nabla / L$ and

$$
\phi_{\mathrm{p}} \frac{\partial c_{\mathrm{p}}}{\partial t}+\left(1-\phi_{\mathrm{p}}\right) \rho_{\mathrm{p}} \frac{\partial q}{\partial t}=\frac{D_{\mathrm{p}}}{r_{\mathrm{p}}^{2}} \frac{\partial}{\partial r_{\mathrm{p}}}\left(r_{\mathrm{p}}^{2} \frac{\partial c_{\mathrm{p}}}{\partial r_{\mathrm{p}}}\right),
$$

where $c_{\mathrm{p}}$ is the concentration within the particle, and $\phi_{\mathrm{p}}$ and $\rho_{\mathrm{p}}$ are the porosity and density of the particles, respectively. The diffusivity of $A s$ inside the particle is $D_{\mathrm{p}}$ and $q$ is the adsorption capacity (kg per $\mathrm{kg}$ of adsorbent). Eq. (5) must be solved at every position $(r, z)$ in the porous domain and so an extra lengthscale to the problem.

where $k_{1,2}$ are the adsorption and desorption rate constants, respectively, and $q_{\mathrm{m}}$ is the maximum amount of contaminant $(\mathrm{kg})$ that can be adsorbed per $\mathrm{kg}$ of material. This equation is closed by applying the initial condition

$$
q=0 \quad \text { at } \quad t=0 .
$$

$$
\begin{aligned}
& \bar{r}=\frac{r}{L}, \quad \bar{r}_{\mathrm{p}}=\frac{r_{\mathrm{p}}}{a_{\mathrm{p}}}, \quad \bar{z}=\frac{z}{L}, \quad \bar{R}=\frac{R}{L}, \quad \bar{t}=\frac{t D_{\mathrm{p}}}{L^{2}}, \\
& \bar{c}=\frac{c}{c_{\mathrm{f}}}, \quad \bar{c}_{\mathrm{p}}=\frac{c_{\mathrm{p}}}{c_{\mathrm{f}}}, \quad \bar{q}=\frac{q}{q_{\mathrm{m}}}, \quad \overline{\boldsymbol{u}}=\boldsymbol{u} / v_{\mathrm{i}}, \quad \bar{p}=p_{a}+\frac{\kappa}{\mu v_{\mathrm{i}} L} p .
\end{aligned}
$$

(ii) At the centre of the particle, $r_{\mathrm{p}}=0$, we apply a symmetry condition, $\partial c_{\mathrm{p}} / \partial r_{\mathrm{p}}=0$.

(iii) Initially the particles do not contain any $A s$, so $c_{\mathrm{p}}=0$ at $t=0$ for $0 \leq r_{\mathrm{p}} \leq a_{\mathrm{p}}$.

Typical parameter values may be found in Table 1.$$
D a=\frac{\kappa}{L^{2}},
$$ 
Table 1: Properties of the physical variables and system parameters

\begin{tabular}{|l|l|l|}
\hline Properties of the adsorbent & Raw laterite & Treated laterite \\
\hline Maximum adsorption capacity, $q_{\mathrm{m}}(\mathrm{kg} / \mathrm{kg})$ & $1.4 \times 10^{-4}[6]$ & $0.0128[12]$ \\
\hline Forward rate constant, $k_{1}\left(\mathrm{~m}^{3} / \mathrm{kg} . \mathrm{s}\right)$ & $2.21 \times 10^{-2}$ & $1.44 \times 10^{-5}$ \\
\hline Backward rate constant, $k_{2}(1 / \mathrm{s})$ & $3.12 \times 10^{-6}$ & $1.23 \times 10^{-6}$ \\
\hline Average density of adsorbent, $\rho_{\mathrm{p}}\left(\mathrm{kg} / \mathrm{m}^{3}\right)$ & $1325[6]$ & $1050[12]$ \\
\hline Average particle radius, $a_{\mathrm{p}}(\mathrm{mm})$ & $2.5[6]$ & $0.25[12]$ \\
\hline Permeability of adsorbent bed, $\kappa\left(\mathrm{m}^{2}\right)$ & $2.9 \times 10^{-8}$ & $1.4 \times 10^{-8}$ \\
\hline Average bed porosity, $\phi$ & $0.48[6]$ & $0.32[12]$ \\
\hline Particle porosity, $\phi_{\mathrm{p}}$ & $0.03[6]$ & $0.39[12]$ \\
\hline Diffusivity of $A s$ through the medium & $\left.D_{l, R L}\right)$ & $D_{l, T L}$ \\
and theoretical prediction from Section $4.1\left(\mathrm{~m}^{2} / \mathrm{s}\right)$ & $3.7 \times 10^{-10}$ & $2.7 \times 10^{-11}$ \\
\hline Diffusivity of $A s$ inside the adsorbent particle & $D_{p, R L}$ & $D_{p, T L}$ \\
\hline and theoretical prediction from Section $4.1\left(\mathrm{~m}^{2} / \mathrm{s}\right)$ & $9.2 \times 10^{-10}$ & $1.4 \times 10^{-10}$ \\
\hline Properties of the liquid & Value & \\
\hline Density of liquid, $\rho_{\mathrm{l}}\left(\mathrm{kg} / \mathrm{m}^{3}\right)$ & 1000 & \\
\hline Viscosity of contaminated water, $\mu(\mathrm{Pa} \mathrm{s})$ & $8.94 \times 10^{-4}$ & \\
\hline Temperature $\left({ }^{\circ} \mathrm{C}\right)$ & 25 & \\
\hline
\end{tabular}

$$
\begin{array}{lr}
\bar{u}=0 \text { and } \bar{v}=1, & \text { at } \bar{z}=0, \\
\bar{u}=\bar{v}=0, & \text { at } \bar{r}=\bar{R}, \\
\bar{u}=\frac{\partial \bar{v}}{\partial \bar{r}}=0, & \text { at } \bar{r}=0, \\
\bar{p}=0, & \text { at } \bar{z}=1, \\
\bar{u}=\bar{v}=0, & \text { at } \bar{t}=0 .
\end{array}
$$

The dimensionless form of Eq. (3) becomes

$$
\frac{\alpha}{P e} \frac{\partial \bar{c}}{\partial \bar{t}}+\bar{u} \frac{\partial \bar{c}}{\partial \bar{r}}+\bar{v} \frac{\partial \bar{c}}{\partial \bar{z}}=\frac{1}{P e}\left(\frac{\partial^{2} \bar{c}}{\partial \bar{z}^{2}}+\frac{1}{\bar{r}} \frac{\partial}{\partial \bar{r}}\left(\bar{r} \frac{\partial \bar{c}}{\partial \bar{r}}\right)\right)-\mathcal{A}\left(\bar{c}-\left.\bar{c}_{\mathrm{p}}\right|_{\bar{r}_{\mathrm{p}}=1}\right),
$$

where

$$
\begin{aligned}
P e & =\frac{v_{\mathrm{i}} L}{D_{\mathrm{l}}}, \\
\alpha & =\phi \frac{D_{\mathrm{p}}}{D_{\mathrm{l}}}, \\
\mathcal{A} & =\frac{3 k_{\mathrm{f}}(1-\phi) L}{v_{\mathrm{i}} a_{\mathrm{p}}}=\frac{3 k_{\mathrm{f}} M}{Q_{\mathrm{i}} \rho_{\mathrm{p}} a_{\mathrm{p}}},
\end{aligned}
$$


Table 2: Typical system properties of the bed in the lab-scale experiments.

\begin{tabular}{|l|l|}
\hline System properties & Value \\
\hline Average inlet concentration of $A s, c_{\mathrm{f}}(\mu \mathrm{g} / \mathrm{L})$ & 80 \\
\hline Average volumetric inflow rate, $Q_{0}(\mathrm{~L} /$ day $)$ & 40 \\
\hline Linear inflow rate, $v_{\mathrm{i}}(\mathrm{m} / \mathrm{s})$ & $3.044 \times 10^{-4}$ \\
\hline Filter radius, $R(\mathrm{~m})$ & 0.022 \\
\hline Filter length, $L(\mathrm{~m})$ & 0.20 \\
\hline
\end{tabular}

\begin{tabular}{|c|c|c|c|}
\hline System properties & Experiment 1 & Experiment 2 & Experiment 3 \\
\hline Material used & RL & $\mathrm{TL}$ & $\mathrm{RL}(0.1 \mathrm{~m})$ on top of TL $(0.1 \mathrm{~m})$ \\
\hline$R e$ & 68.1 & 68.1 & 68.1 (for both layers) \\
\hline$R e_{\mathrm{p}}$ & 0.85 & 0.085 & 0.85 (top), 0.085 (bottom) \\
\hline $\mathrm{Pe}$ & $1.64 \times 10^{5}$ & $2.25 \times 10^{6}$ & $1.64 \times 10^{5}($ top $), 2.25 \times 10^{6}($ bottom $)$ \\
\hline$D a$ & $7.25 \times 10^{-7}$ & $3.5 \times 10^{-7}$ & $7.25 \times 10^{-7}$ (top), $3.5 \times 10^{-7}$ (bottom) \\
\hline$S c_{\mathrm{p}}$ & $0.97 \times 10^{3}$ & $6.38 \times 10^{3}$ & $0.97 \times 10^{3}($ top $), 6.38 \times 10^{3}($ bottom $)$ \\
\hline$S h$ & 8.1 & 4.7 & 8.1 (top), 4.7 (bottom) \\
\hline$\beta_{1}$ & 76.9 & 0.33 & 76.9 (top layer, RL), 0.33 (bottom) \\
\hline$\beta_{2}$ & 135.8 & 374 & 135.8 (top), 374 (bottom) \\
\hline $\mathcal{A}$ & 6.40 & 12.4 & 6.40 (top), 12.4 (bottom) \\
\hline $\mathcal{B}$ & 0.36 & 0.26 & 0.36 (top), 0.26 (bottom) \\
\hline
\end{tabular}

and the mass of adsorbent, $M=\pi R^{2} L \rho_{\mathrm{p}}(1-\phi)$ and $Q_{\mathrm{i}}=\pi R^{2} v_{\mathrm{i}}$ is the volumetric inlet flux. The parameter $P e$ is the Péclet number and is typically large $\left(O\left(10^{5}-10^{6}\right)\right)$ (see Tables 2 and 3 ); $\mathcal{A}$ relates the competition between the macroscale convection and intra-particle dynamics. Here, $\mathcal{A}=6.4-12.4$ as reported in Tables 2 and 3, meaning that both the macroscale and microscale phenomena are important, necessitating analysis at both the scales.

The associated dimensionless initial and boundary conditions are

$$
\begin{array}{rlrl}
\bar{c} & =0, & \text { at } \bar{t}=0, \\
\bar{c} & =1, & \text { at } \bar{z}=0, \\
\frac{\partial \bar{c}}{\partial \bar{r}}=1, & \text { at } \bar{r}=\bar{R}, \\
\frac{\partial \bar{c}}{\partial \bar{r}}=0, & \text { at } \bar{r}=0, \\
\frac{\partial \bar{c}}{\partial \bar{z}}=1, & \text { at } \bar{z}=1 .
\end{array}
$$

Since $P e \gg \mathcal{A}$ and $\alpha$ is also expected to be $O(1)$, the transient term in Eq. (14) can be neglected, to give

$$
\bar{u} \frac{\partial \bar{c}}{\partial \bar{r}}+\bar{v} \frac{\partial \bar{c}}{\partial \bar{z}}=\frac{1}{P e}\left(\frac{\partial^{2} \bar{c}}{\partial \bar{z}^{2}}+\frac{1}{\bar{r}} \frac{\partial}{\partial \bar{r}}\left(\bar{r} \frac{\partial \bar{c}}{\partial \bar{r}}\right)\right)-\mathcal{A}\left(\bar{c}-\left.\bar{c}_{\mathrm{p}}\right|_{\bar{r}_{\mathrm{p}}=1}\right) .
$$

There will be a short transient start-up period that will not be captured by this simplified system. We note that in the bulk we would also expect the diffusive terms that are premultiplied by $1 / P e$ to be negligible too, further simplifying this equation, but these will become important near the boundaries 
Table 3: Typical system properties for the field-scale experiments composed of TL as the active As removal material.

\begin{tabular}{|l|l|}
\hline System properties & Value \\
\hline Inlet concentration of $A s, c_{\mathrm{f}}(\mu \mathrm{g} / \mathrm{L})$ & 100 \\
\hline Volumetric inflow rate, $Q_{0}(\mathrm{~L} /$ day $)$ & 100 \\
\hline Linear inflow rate, $v_{\mathrm{i}}(\mathrm{m} / \mathrm{s})$ & $3.68 \times 10^{-5}$ \\
\hline Radius, $R(\mathrm{~m})$ & 0.10 \\
\hline Length of laterite region, $L(\mathrm{~m})$ & 0.35 \\
\hline Length of the whole filter $(\mathrm{m})$ & 1.0 \\
\hline$R e$ & 14.42 \\
\hline$\left.R e_{\mathrm{p}}\right)$ & 0.01 \\
\hline$P e$ & $4.77 \times 10^{5}$ \\
\hline$D a$ & $1.14 \times 10^{-7}$ \\
\hline$S h$ & 2.94 \\
\hline$\beta_{1}$ & 1.26 \\
\hline$\beta_{2}$ & 1145.5 \\
\hline $\mathcal{A}$ & 22.0 \\
\hline $\mathcal{B}$ & 0.068 \\
\hline
\end{tabular}

and are required to satisfy the boundary conditions here, which must be satisfied for all time and so we retain them for our numerical computation.

The dimensionless version of the intra-particle dynamic equation, (Eq. 5), is

$$
\left(\frac{a_{\mathrm{p}}}{L}\right)^{2} \phi_{\mathrm{p}} \frac{\partial \overline{c_{\mathrm{p}}}}{\partial \bar{t}}+\left(1-\phi_{\mathrm{p}}\right) \mathcal{B} \frac{\partial \bar{q}}{\partial \bar{t}}=\frac{1}{\bar{r}_{\mathrm{p}}^{2}} \frac{\partial}{\partial \bar{r}_{\mathrm{p}}}\left(\bar{r}_{\mathrm{p}}^{2} \frac{\partial \bar{c}_{\mathrm{p}}}{\partial \bar{r}_{\mathrm{p}}}\right)
$$

where

$$
\mathcal{B}=\frac{\rho_{\mathrm{p}} a_{\mathrm{p}}^{2} q_{\mathrm{m}}}{c_{\mathrm{f}} L^{2}}
$$

which quantifies the ratio of intraparticle diffusion to adsorption. In this set-up, $\mathcal{B}=0.26-0.36$, as observed from Tables 2 and 3. This signifies that these two intra-particle phenomena are comparable and are important and emphasises the need to study the full-scale intra-particle dynamics.

Since, $a_{\mathrm{p}} / L \ll 1$ (see Tables 1 and 2 ), the temporal term $\partial \overline{c_{\mathrm{p}}} / \partial \bar{t}$ in Eq. (20) can be ignored, leaving

$$
\left(1-\phi_{\mathrm{p}}\right) \mathcal{B} \frac{\partial \bar{q}}{\partial \bar{t}}=\frac{1}{\bar{r}_{\mathrm{p}}^{2}} \frac{\partial}{\partial \bar{r}_{\mathrm{p}}}\left(\bar{r}_{\mathrm{p}}^{2} \frac{\partial \bar{c}_{\mathrm{p}}}{\partial \bar{r}_{\mathrm{p}}}\right) .
$$

The dimensionless boundary and initial conditions are

$$
\begin{aligned}
S h\left(\bar{c}-\left.\bar{c}_{\mathrm{p}}\right|_{\bar{r}_{\mathrm{p}}=1}\right) & =\phi_{\mathrm{p}} \frac{\partial \bar{c}_{\mathrm{p}}}{\partial \bar{r}_{\mathrm{p}}}, & & \text { at } \quad \bar{r}_{\mathrm{p}}=1, \\
\frac{\partial \bar{c}_{\mathrm{p}}}{\partial \bar{r}_{\mathrm{p}}} & =0, & & \text { at } \quad \bar{r}_{\mathrm{p}}=0, \\
\bar{c}_{\mathrm{p}} & =0, & & \text { at } \quad \bar{t}=0,
\end{aligned}
$$

where $S h=k_{\mathrm{f}} a_{\mathrm{p}} / D_{\mathrm{p}}=f\left(R e_{\mathrm{p}}, S c_{\mathrm{p}}\right)$ is the Sherwood number. For suitably low flow rates (when the Reynolds number, $R e=\rho_{\mathrm{l}} v_{\mathrm{i}} L / \mu \leq 250$, where $\rho_{\mathrm{l}}$ is the fluid density, which is true in our case as seen in 
Table 2) around small spherical particles (of around a few $\mathrm{mm}$ or smaller), the mass-transfer coefficient (in terms of $S h$ ) may be expressed in terms of known variables as [16]

$$
S h=2+0.5 R e_{\mathrm{p}}^{1 / 2} S c_{\mathrm{p}}^{1 / 3},
$$

where $R e_{\mathrm{p}}=\rho_{\mathrm{l}} v_{\mathrm{i}} a_{\mathrm{p}} / \mu$ and $S c_{\mathrm{p}}=\mu / \rho_{\mathrm{l}} D_{\mathrm{p}}$ are the particle Reynolds number and Schmidt number, respectively. For larger particles other relationships of similar form have been established - see, for example, [17].) The parameter $\mathcal{A}$ defined in Eq. (17) may be expressed in terms of $S h$ rather than $k_{\mathrm{f}}$ as $\mathcal{A}=3 S h D_{\mathrm{p}}(1-\phi) L / a_{\mathrm{p}}^{2} v_{\mathrm{i}}$, which, combined with Eq. (24) allows us to determine $\mathcal{A}$. The adsorptionkinetic equation, Eq. (7), is scaled as

$$
\frac{\partial \bar{q}}{\partial \bar{t}}=\beta_{1} \bar{c}_{\mathrm{p}}(1-\bar{q})-\beta_{2} \bar{q},
$$

where $\beta_{1}=k_{1} c_{\mathrm{f}} L^{2} / D_{\mathrm{p}}$ and $\beta_{2}=k_{2} L^{2} / D_{\mathrm{p}}$ are the dimensionless adsorption and desorption rates, while the initial condition (8) becomes

$$
\bar{q}=0 \quad \text { at } \quad \bar{t}=0,
$$

to close the system. Eq. (19) is coupled with the intra-particle mass transport through Eq. (22) at all $(r, z)$ and the hydrodynamics through Eqs. (10) and (11), all of which are solved together using the appropriate initial and boundary conditions.

\subsection{Quantity of interest}

The principal quantity of interest is the average concentration of the contaminant at the outlet,

$$
\bar{c}_{\text {avg }}(\bar{t})=\left.\frac{2}{\bar{R}^{2}} \int_{0}^{\bar{R}} \bar{r} \bar{c}\right|_{\bar{z}=1} \mathrm{~d} \bar{r} .
$$

During filtration, eventually the soil bed reaches its maximum adsorption capacity, termed breakthrough. Then, the concentration at the outlet is equal to the concentration at the inlet. Mathematically this is equivalent to the time at which $\bar{c}(\bar{r}, \bar{z}, \bar{t})=1$ everywhere in the filter, at which point the filter is completely saturated (or exhausted).

\section{Materials and methods}

\subsection{Laterite material}

Laterite is a type of soil rich in iron, silica and aluminium found abundantly in hot and wet tropical areas. The quality of the laterite soil as adsorbent for arsenic can be quantified by degree of laterization defined as the silica-sesquioxide (S-S) ratio [18]. The smaller the S-S ratio, the higher is the degree of laterization. The laterite used in this work was taken from Kharagpur $\left(22.346^{\circ} \mathrm{N}, 87.2320^{\circ} \mathrm{S}\right)$ having an S-S ratio of 0.42 .

Raw laterite is treated with hydrochloric acid $(6 \mathrm{~N})$ in the ratio $50 \mathrm{~g}$ laterite to $200 \mathrm{ml}$ of acid at $70^{\circ} \mathrm{C}$ for 2 hours followed by the addition of $4 \mathrm{~N}$ sodium hydroxide continuously until the $\mathrm{pH}$ reached 8.5. The acid-alkali treatment was carried out under continuous stirring (500 rpm) after which the treated material was washed using tap water until $\mathrm{pH}$ of the wash water reached $6.5 \pm 0.5$ and was air dried [8]. 


\subsection{Adsorption isotherm}

The adsorption isotherm for the soil-bed material, given by the steady-state version of Eq. (7), was measured by conducting batch experiments with either raw laterite (RL) or treated laterite (TL). Synthetic solutions of $A s$ in water with concentrations ranging from $10 \mathrm{mg} / \mathrm{L}$ to $1000 \mathrm{mg} / \mathrm{L}$ were prepared using sodium arsenate heptahydrate. A fixed quantity of the adsorbent was inserted in a known volume $V$ of synthetic solution and placed in a shaker (speed: $150 \mathrm{rpm}$ temperature: $25^{\circ} \mathrm{C}$ ). After 24 hours, the adsorbent was filtered and the residual concentration of $A s$ was measured using an atomic adsorption spectrophotometer (Analyst 700 coupled with MHS-15, PerkinElmer Instruments, USA). The amount of As adsorbed, $q_{\mathrm{e}}$, was then calculated using a standardised method employing the dimensional equation expressing conservation of mass:

$$
q_{\mathrm{e}}=\frac{\left(c_{0}-c_{\mathrm{e}}\right) V}{M}
$$

where $c_{0}$ and $c_{\mathrm{e}}$ are the initial and equilibrium concentrations of arsenic and recall that $M$ denotes the mass of the adsorbent used. This expression was inserted into the Langmuir isotherm, obtained from the steady-state version of Eq. (7), and expressed in linear form,

$$
\frac{c_{\mathrm{e}}}{q_{\mathrm{e}}}=\frac{1}{q_{\mathrm{m}}} c_{\mathrm{e}}+\frac{1}{K_{0} q_{\mathrm{m}}}
$$

where $K_{0}=k_{1} / k_{2}$ is the adsorption equilibrium constant. Comparing the linear plot with the data allows us to extract the value for $q_{\mathrm{m}}$ (through the gradient) and $K_{0}$ (through the intercept), with values given in Table 1.

\subsection{Kinetic study}

The constant $k_{1}$ that appears in Eq. (7) was determined by conducting adsorption experiments using a feed solution of volume $V$ and adsorbent of mass $M$ using a fixed arsenic concentration of $100 \mathrm{mg} / \mathrm{L}$ in the feed. Assuming the concentration in the filter, $c$, was constant throughout, this was related to the total adsorbed amount via the mass-balance relationship

$$
q=\frac{\left(c_{0}-c\right) V}{M}
$$

Rearranging Eq. (30) for $c$ and inserting into Eq. (7) gives the expression

$$
\frac{\partial q}{\partial t}=k_{1}\left(c_{0} q_{\mathrm{m}}-c_{0} q-\frac{q_{\mathrm{m}} q M}{V}+\frac{M q^{2}}{V}-\frac{q}{K_{0}}\right) .
$$

Plotting the experimental values for the left-hand side of (31) versus the bracketed term on the righthand side determines $k_{1}$ as the corresponding gradient; $k_{2}$ is then determined from $K_{0}=k_{1} / k_{2}$, with values given in Table 1.

\subsection{Measurement of bed permeability}

The properties of the adsorbent bed in the experimental set-up are provided in Table 1. The bed permeability, $\kappa\left(\mathrm{m}^{2}\right)$, was measured by passing distilled water through the bed in the upward direction at varying flow rates $Q$ (from 1 to $20 \mathrm{~L} / \mathrm{h}$ ). The flow rate was controlled by using a regulated DC power supply (Aplab, model: L1602) for the pump; the error is within 2\%. The pressure drop across the 
bed, $\Delta p$, was measured using a mercury manometer and plotted against the corresponding flow rate.

The permeability was then extracted using Darcy's law, which follows from Eq. (2) for a unidirectional spatially uniform velocity,

$$
\frac{Q}{A}=-\frac{\kappa}{\mu} \frac{\Delta p}{L}
$$

where $A=\pi R^{2}$ is the cross-sectional area of the filter, $\mu$ is the viscosity of water and $L$ is the column length. The value found for the permeability $\kappa$ is given in Table 1 .

\subsection{Column studies}

For the lab-scale study, a cylindrical column of inner radius $0.022 \mathrm{~m}$ was used with a wire mesh at the bottom to prevent loss of adsorbent. An overhead tank was filled with synthetic solution of $A s$ of a specified concentration and the flow rate was adjusted according to the requirements. The flow rate was controlled using a pinch-cock to get the desired throughput, which is reasonably accurate having a variation of $\pm 5 \%$ of the mean value. The flow rate is monitored almost every 6 hours to check for the variability from its preset value. The valve (pinch-cock) opening is adjusted accordingly to ensure the desired flow rate as required. The feed and permeate samples were analysed using an atomic absorption spectrophotometer. Different experiments were conducted varying the feed flow rates, feed $A s$ concentration and the bed height. The specifications of the column study along with operating conditions are presented in Table 2 .

\section{Model validation}

There are two filters of interest: lab-scale filters and field-scale filters. Lab-scale filters are used to study the system behaviour in a controlled environment; field-scale filters are used in practical scenarios to supply drinking water domestically, in schools, or in communities, and hence they are typically larger (see Table 3). Field-scale filters also consist of layers of different porous materials, each designed to remove a specific contaminant. The TL section of a field-scale filter occupies around $0.35 \mathrm{~m}$ of the entire filter height of around $1 \mathrm{~m}$, with the remaining layers having no As adsorption capacity. A photograph of the field-scale filter is shown in Fig. 1 while the lab-scale filter is shown in Fig. 2. The typical dimensions of these filters are presented in Table 2.

We first determine the diffusion coefficients $\left(D_{1}\right.$ and $\left.D_{\mathrm{p}}\right)$ and validate our model with two different experiments using RL and TL as the adsorbent, referred to as experiments 1 and 2 respectively in Table 2 . All system parameters are then known. The model is then used to predict the filter performance under varying operating conditions and is compared with the experimental results. Following the validation, the model is used to probe the inter- and intra-particle dynamics that cannot be extracted from the experiments (Sections 5.1 and 5.2). Finally, the model is used to predict the lifetime of the field unit and how it may be used as a tool for determining the required filter for a given challenge. This is the main purpose, allowing predictions to be made that would take many years to find experimentally. 


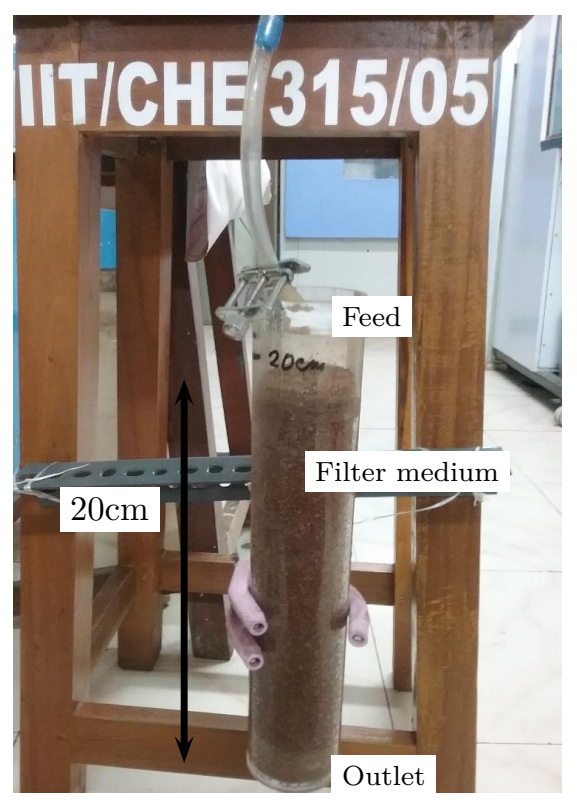

Figure 2: Photograph of a lab-scale filter.

\subsection{Parameter estimation}

We solve the system of equations (10), (11), (19), (22) and (25), subject to the initial and boundary conditions (13), (18), (23) and (26) with the dimensionless relation (24), using the finite-element method package COMSOL v5.4 ${ }^{\circledR}$ for the values listed in Tables 1 and 2.

A synthetic solution of $A s$ with concentration $80 \mu \mathrm{g} / \mathrm{L}$ was introduced into the lab-scale filter at a flow rate of $40 \mathrm{~L} /$ day $\left(v_{\mathrm{i}}=3.044 \times 10^{-4} \mathrm{~m} / \mathrm{s}\right)$. The diffusion coefficients, $D_{1}$ and $D_{\mathrm{p}}$ were then determined by fitting the model to the experimental measurements for $\bar{c}_{\text {avg }}$ at the filter outlet for a single-layer lab-scale filter using least-squares fitting (see Figs. 3a and b).

The goodness of fit was verified using the linear regression coefficient, $\mathcal{R}^{2}$, with strong correlation values of 0.97 for RL and 0.98 for TL [19]. The result of this comparison gives $D_{1, \mathrm{RL}}=3.7 \times 10^{-10} \mathrm{~m}^{2} / \mathrm{s}$, $D_{\mathrm{p}, \mathrm{RL}}=9.2 \times 10^{-10} \mathrm{~m}^{2} / \mathrm{s}$ for RL, while $D_{\mathrm{l}, \mathrm{TL}}=2.7 \times 10^{-11} \mathrm{~m}^{2} / \mathrm{s}, D_{\mathrm{p}, \mathrm{TL}}=1.4 \times 10^{-10} \mathrm{~m}^{2} / \mathrm{s}$ are obtained for TL. We attribute the lower diffusivity in the treated laterite than in the raw laterite to the lower porosity, which impedes the spread of As. Within the pores themselves, the As has a much lower diffusivity in the treated laterite than the raw laterite despite having a much higher porosity, which has been reported elsewhere [20]. This is thought to be due to the channeling structure within the raw laterite pore, which increases the mean free path.

With the parameters $D_{\mathrm{l}}$ and $D_{\mathrm{p}}$ determined all system parameters are now known and the model can be used in a fully predictive manner to determine the behaviour in other scenarios. To verify the predictive power of the model, a series of further simulations were conducted and compared with experimental data. First, the filtration behaviour of a filter having a single layer of volumes of RL followed by TL, each occupying a height of $L / 2$ was considered (Fig. 3c). In this case, no fitting parameters remained. The $\mathcal{R}^{2}$ value for this system was 0.973 , indicating that the model captures the data well. The effect of varying the feed flow rate, contaminant concentration, and bed height was then studied (Fig. 4) and all exhibited a good fit. This confirms that the model suitably represents the working system and can be 
$\bar{t}$
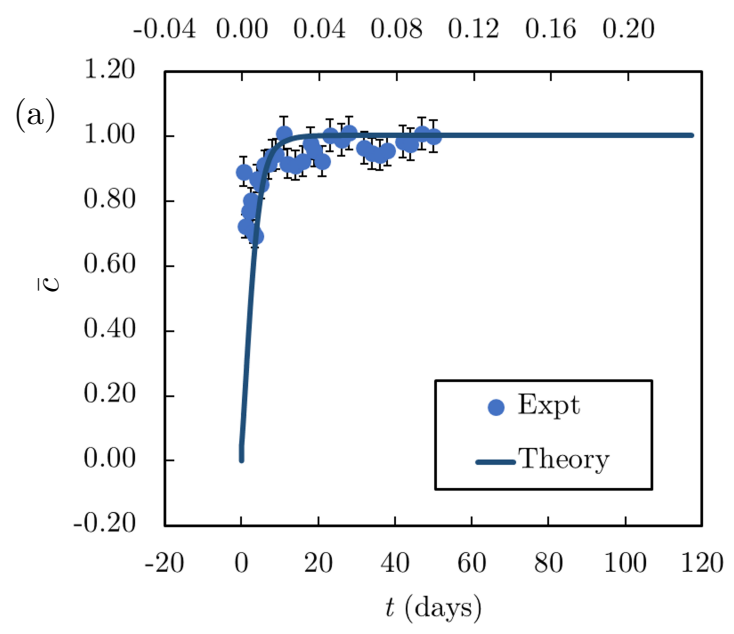

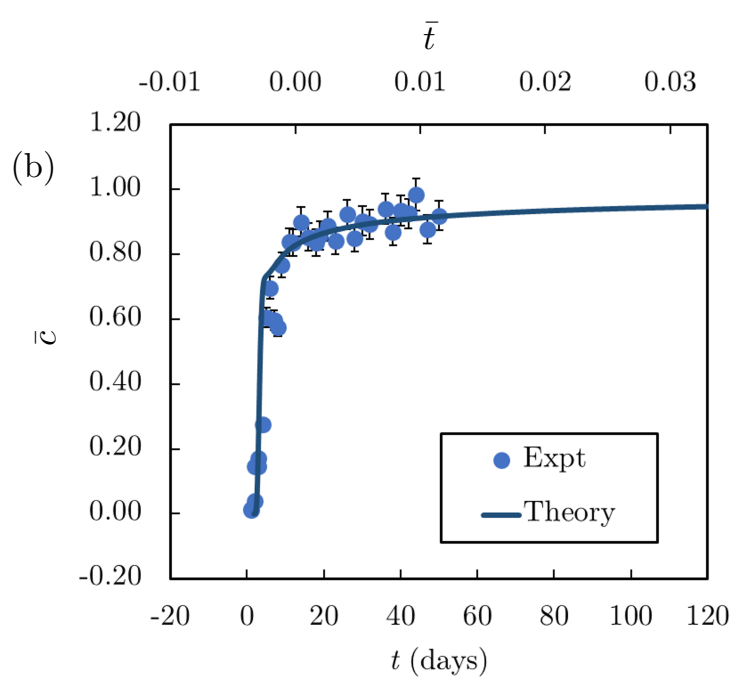

(b)

$\bar{t}$

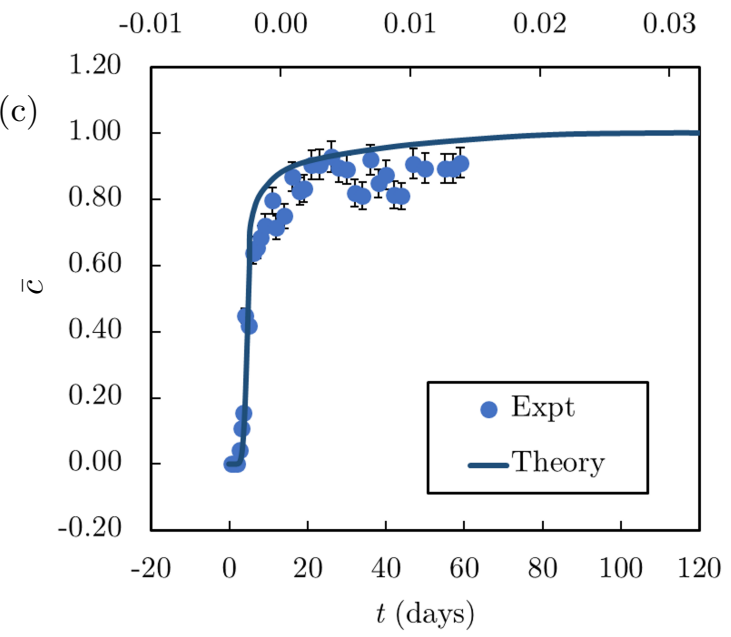

Figure 3: Comparison of the experimental observation with the model simulation using (a) RL as the adsorbent (experiment 1 in Table 2); (b) TL as the adsorbent (experiment 2 in Table 2) and (c) a dual-layer bed of RL (0.1m) followed by TL bed of $0.1 \mathrm{~m}$ (experiment 3 in Table 2). No fitting parameters are used in (c), supporting the validity of the model. The system conditions are listed in Tables 1 and 2 . The error bars represent $\pm 5 \%$ of the mean experimental value. 

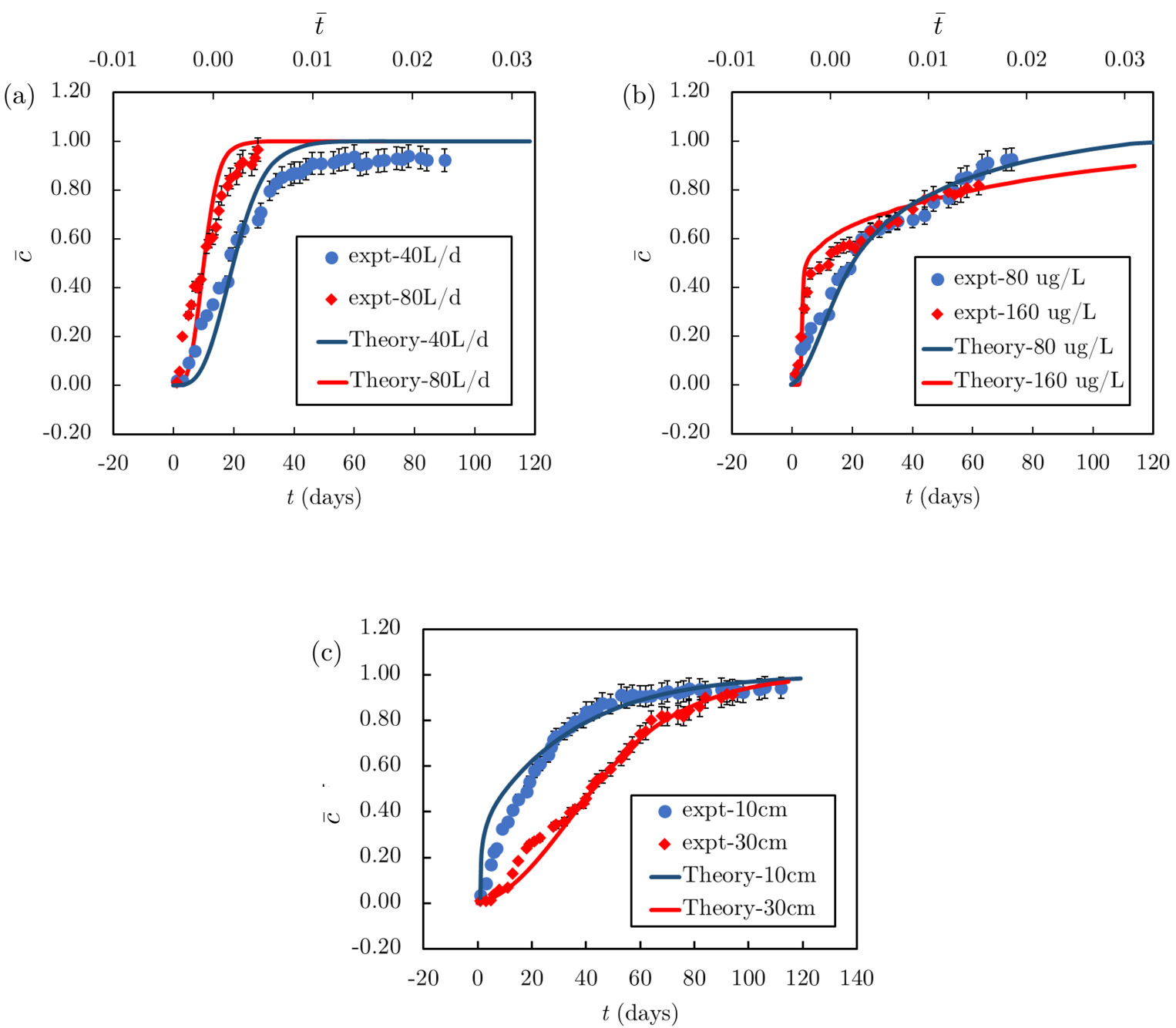

Figure 4: Effect of changing the system conditions on the breakthrough profiles. Variation in (a) the feed flow rate as 40 and $80 \mathrm{~L} / \mathrm{d}$, (b) the feed As concentration as 80 and $160 \mu \mathrm{g} / \mathrm{L}$, and (c) the bed height as 10 and $30 \mathrm{~cm}$. The symbols represent the experimental data while the solid line is the model prediction. The error bars represent $\pm 5 \%$ of the mean values obtained experimentally. The values of the remaining parameters for the experimental condition and the model calculations are listed in Tables 1 and 2. The dimensionless scale for time is shown on the upper $x$-axis in (a) and (b). Since the dimensionless time depends on bed height this differs for the two curves displayed in (c) and so is not shown in this graph.

\section{Model analysis}

In this section we now use the model to probe the system behaviour more deeply. Specifically, we first explore the inter and intra-particle dynamics in Sections 5.1 and 5.2 respectively. We then turn our attention to the key challenge of using our model in a predictive way to determine the lifetime of the filter and its dependence on the filter parameters in Section 5.3. 


\subsection{Inter-particle dynamics}

The fluid is injected at the top of the filter and the flow is expected to become unidirectional after a short transient period. Assuming $\overline{\boldsymbol{u}}=\bar{v}(\bar{r}, \bar{z}, \bar{t}) \boldsymbol{e}_{\boldsymbol{z}}$, Eq. (10) immediately indicates that $\bar{v}$ is independent of $\bar{z}$. The radial component of Eq. (11) then gives $\bar{p}=\bar{p}(\bar{z}, \bar{t})$, while the axial component reads

$$
-\frac{\partial \bar{p}}{\partial \bar{z}}+\frac{D a}{\phi \bar{r}} \frac{\partial}{\partial \bar{r}}\left(\bar{r} \frac{\partial \bar{v}}{\partial \bar{r}}\right)-\bar{v}=0
$$

Eq. (33) possesses an analytic solution, which, upon application of the symmetry boundary conditions $\partial \bar{v} / \partial \bar{r}=0$ on $\bar{r}=0$ and the no-slip condition $\bar{v}=0$ on $\bar{r}=\bar{R}$, gives

$$
\bar{v}=\left[\frac{I_{0}\left(\sqrt{\frac{\phi}{D a}} \bar{r}\right)}{I_{0}\left(\sqrt{\frac{\phi}{D a}} \bar{R}\right)}-1\right] \frac{\partial \bar{p}}{\partial \bar{z}},
$$

where $I_{n}$ denotes the modified Bessel function of the first kind, with $n=0$ in this case. Application of the dimensionless inlet flux condition in integrated form,

$$
\int_{0}^{\bar{R}} \bar{r} \bar{v} \mathrm{~d} \bar{r}=\frac{1}{2} \bar{R}^{2},
$$

provides the expression for the fluid pressure after integrating with respect to $\bar{z}$ and applying the outlet condition $(13 d)$,

$$
\bar{p}=\frac{(1-\bar{z}) I_{0}\left(\sqrt{\frac{\phi}{D a}} \bar{R}\right)}{I_{2}\left(\sqrt{\frac{\phi}{D a}} \bar{R}\right)} .
$$

We compare the axial velocity profile (34) with the numerical solution in Fig. 5(a) as we vary the filter aspect ratio $\bar{R}$ and find that the two solutions agree well. For smaller values of $\bar{R}$, a dependence of the axial velocity on depth $\bar{z}$ emerges (Fig. 5b), which is consistent with the agreement in $\bar{v}(r)$ for larger values of $\bar{R}$ (Fig. 5a). This indicates that Eq. (34) provides a good approximation to the flow for filters whose aspect ratio is not too small.

\subsection{Intra-particle dynamics}

An asset of our model is that, in addition to modelling the inter-particle dynamics, we also capture the intra-particle behaviour, which is difficult to observe experimentally. Studying the As concentration inside the particle, we find that, for particles at the inlet, $(\bar{r}, \bar{z})=(0,0)$, at the particle surface $\bar{r}_{\mathrm{p}}=1$, $\bar{c}_{\mathrm{p}}$ attains 1 almost immediately and remains at this value thereafter (Fig. 6a). However, at the exit, $(\bar{r}, \bar{z})=(0,1)$, the surface concentration $\left(\bar{c}_{\mathrm{p}}\right.$ at $\left.\bar{r}=1\right)$ takes a longer time to approach 1 . Eventually, the intra-particle concentration reaches saturation, but this happens on a much longer timescale than the bulk removal timescale on which we have non-dimensionalised (Fig. 6c). This arises since contaminant is transported by advection and diffusion in the inter-particle space (with advection dominating since $P e \gg$ 1) but occurs solely by diffusion in the intra-particle space. Thus, at the time we term breakthrough, when the inter-particle concentration reaches 1 everywhere in the liquid, the filter will still continue to adsorb arsenic, albeit at a much slower rate, until the intra-particle concentration also reaches 1 everywhere in space. 

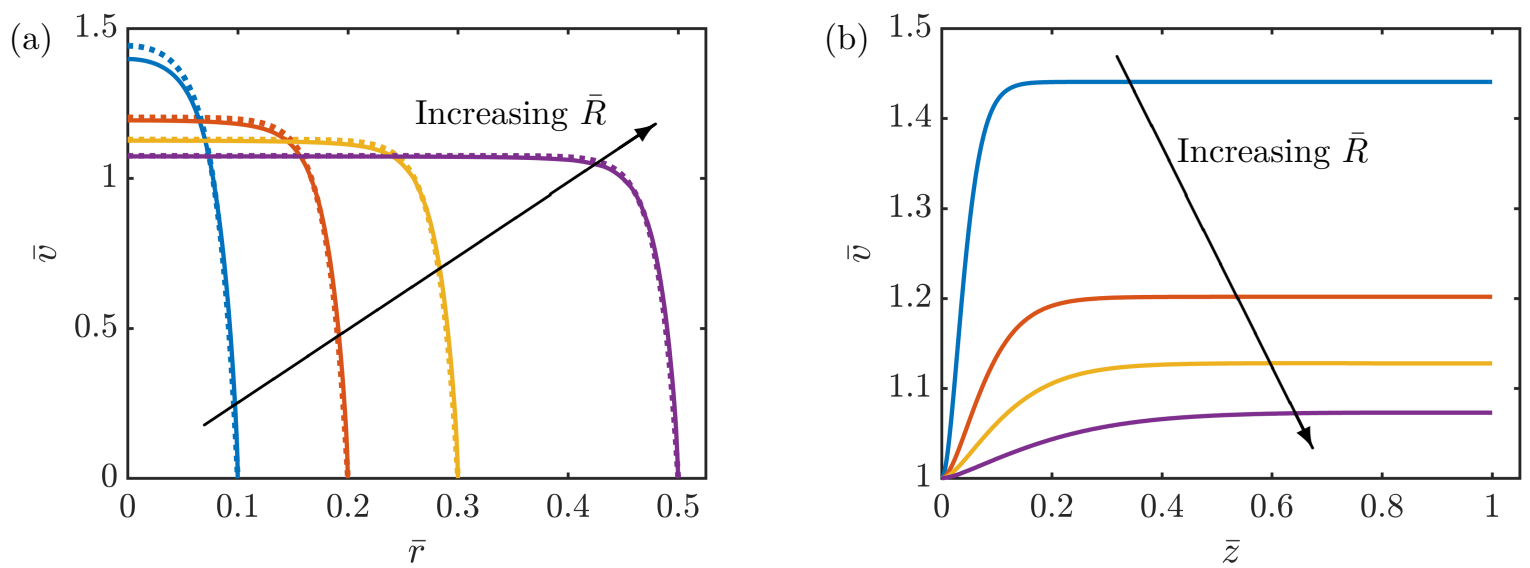

Figure 5: Profile of the fluid velocity $\bar{v}$ as a function of (a) $\bar{r}$, at $\bar{z}=1$ and (b) $\bar{z}$, at $\bar{r}=0$, on increasing the aspect ratio, $\bar{R}=0.1,0.2,0.3$ and 0.5 , in the direction of the arrow. The dotted curves in (a) represent the analytical solution corresponding to Eq. (34). The sample values of $\phi=0.32$ (for a treated-laterite filter) and $L=0.2$, and $D a=10^{-4}$ for illustrative purposes, are used in the calculation. The values of the other parameters are listed in Tables 1 and 2.
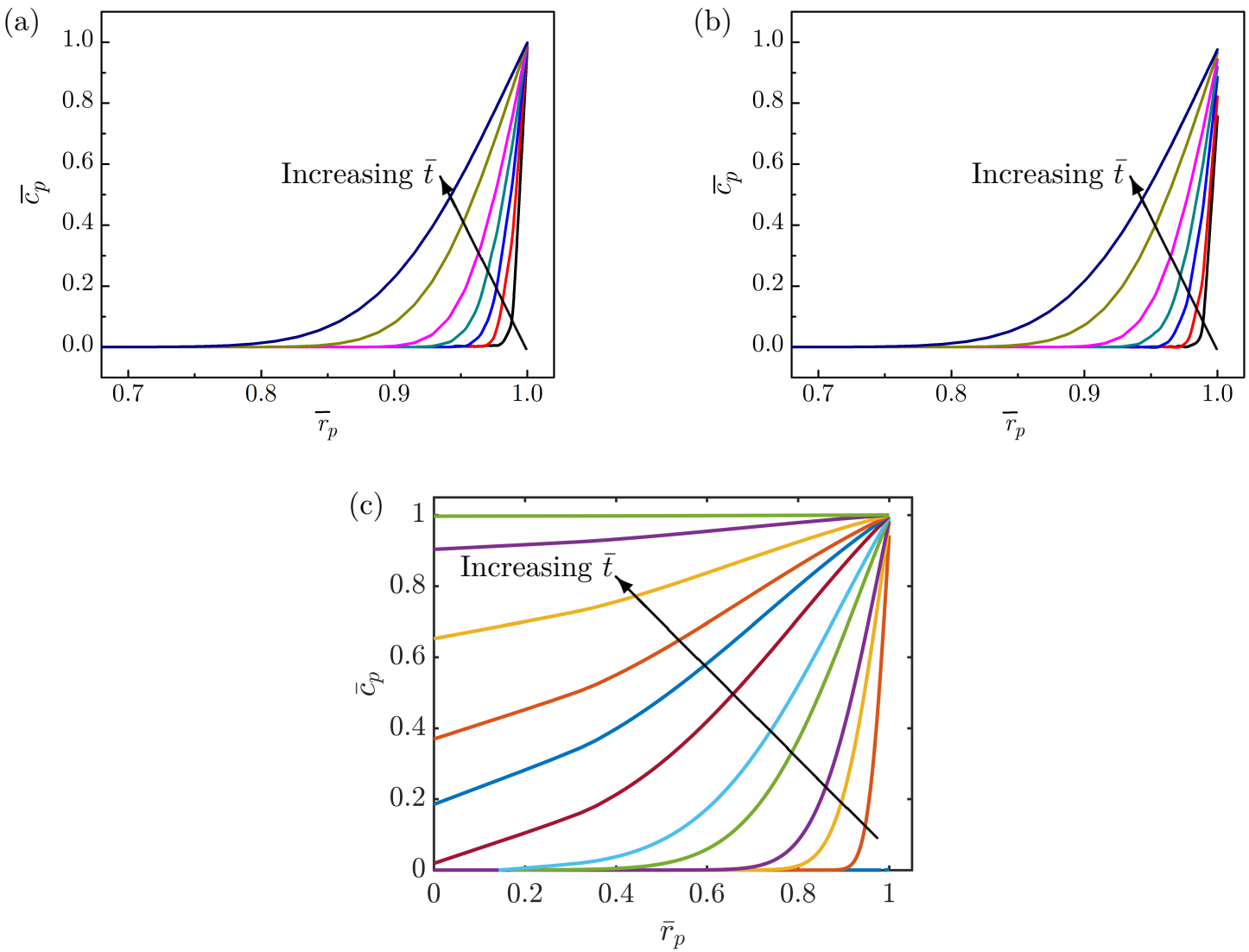

Figure 6: Profile of the intra-particle contaminant $(A s)$ concentration from Experiment $2, \bar{c}_{\mathrm{p}}$ along the particle spherical coordinate with time $\bar{t}$ at (a) $(\bar{r}, \bar{z})=(0,0)$ and (b) $(\bar{r}, \bar{z})=(0,1)$. The arrow indicate the profiles with increasing time, $\bar{t}=$ $0.01,0.02,0.05,0.1,0.2,0.5$ and 1.0. The plot in (c) shows the long-time dynamics inside the particle for $\bar{t}=0.2,1,2,6$, $10,20,30,40,60,100,200$ (in the direction of the arrow), at $(\bar{r}, \bar{z})=(0,0)$. The values of the parameters used correspond to the lab-scale filter, as listed in Tables 1 and 2. 


\subsection{Dependence on filter lifetime on key parameters}

In this final section we use the model for its chief purpose, to determine the lifetime of a filter for a given requirement. The governing system of equations (10), (11), (19), (22) and (25), subject to the initial and boundary conditions (13), (18), (23) and (26) with the dimensionless relation (24) is described by nine dimensionless parameters: $D a, \phi, \phi_{\mathrm{p}}, P e, \mathcal{A}, \mathcal{B}, R e_{\mathrm{p}}, S h, \beta_{1}$ and $\beta_{2}$. It would be an onerous task to study the dependence of the system on each of these parameters individually, but we can facilitate this analysis by recasting the problem in terms of the following parameters:

$$
\mathcal{M}=\frac{M}{\pi R^{2} \rho_{\mathrm{p}}(1-\phi)\left(\frac{a_{\mathrm{p}}^{3} \mu}{\rho_{1} D_{\mathrm{p}}}\right)^{1 / 3}}, \quad \mathcal{Q}_{\mathrm{i}}=\frac{Q_{\mathrm{i}}}{\pi R^{2}\left(\frac{\mu D_{\mathrm{p}}^{2}}{\rho_{1} a_{\mathrm{p}}^{3}}\right)}, \quad \quad \mathcal{C}_{\mathrm{f}}=\frac{c_{\mathrm{f}}}{\left(\frac{\rho_{\mathrm{l}} D_{\mathrm{p}}}{\mu}\right) q_{\mathrm{m}} \rho_{\mathrm{p}}}
$$

These dimensionless parameters encapsulate the dependence on the key variables of interest, namely mass of filter, throughput and feed contaminant concentration, respectively. Expressing the system in this way allows us to perform efficient parameter sweeps to determine how the filter behaviour will change when we vary these key parameters. The nine dimensionless parameters can then be expressed in terms of these three core parameters, along with material parameters that will be unchanged (or can be easily ascertained by experiments):

$$
\begin{gathered}
D a=\left(\frac{a_{\mathrm{p}}^{2} \mu^{2 / 3} \kappa}{\rho_{\mathrm{l}}^{2 / 3} D_{\mathrm{p}}^{2 / 3}}\right) \frac{1}{\mathcal{M}^{2}}, \quad P e=\left(\frac{\mu^{2} D_{\mathrm{p}}}{\rho_{\mathrm{l}}^{2} D_{\mathrm{l}}^{3}}\right)^{1 / 3} \mathcal{Q}_{\mathrm{i}} \mathcal{M}, \quad \mathcal{A}=3(1-\phi) \frac{4+\mathcal{Q}_{\mathrm{i}}^{1 / 2}}{2 \mathcal{Q}_{\mathrm{i}}} \mathcal{M}, \quad \mathcal{B}=\frac{\mathcal{M}}{\mathcal{C}_{\mathrm{f}}}, \\
S h=2+\frac{1}{2} \mathcal{Q}_{\mathrm{i}}^{1 / 2}, \quad \beta_{1}=\frac{k_{1} a_{\mathrm{p}}^{2} q_{\mathrm{m}} \rho_{\mathrm{p}}}{D_{\mathrm{p}}} \mathcal{Q}_{\mathrm{i}} \mathcal{C}_{\mathrm{f}}, \quad \beta_{2}=k_{2} a_{\mathrm{p}}^{2}\left(\frac{\mu^{2}}{\rho_{\mathrm{l}}^{2} D_{\mathrm{p}}^{5}}\right)^{1 / 3} \mathcal{Q}_{\mathrm{i}}^{2},
\end{gathered}
$$

while, $\phi$ and $\phi_{\mathrm{p}}$ are material parameters.

The lifetime $\bar{t}_{1}$ of the filter is defined as the time at which $\bar{c}_{\text {avg }} c_{\mathrm{f}}>10 \mu \mathrm{g} / \mathrm{L}$, according to the WHO guidelines [21]. As expected, the lifetime of the filter decreases with increasing throughput because of the higher processing rate (Fig. 7a). However, it varies in both a nonlinear and non-power-law manner as a result of the coupled intra- and inter-particle dynamics, a trend that would be difficult to anticipate from experiments. Thus, this predicts that when doubling the filter flow rate, the lifetime falls by less than half, which may prove promising for upscaling. An increase in the mass of adsorbent increases the total adsorption capability of the filter and thus increases the lifetime (Fig. 7a). Increasing the feed concentration leads to a lower filter lifetime, but this can be countered by increasing the mass of adsorbent used (Fig. 7b). As with the relationship between filter lifetime and throughput, the dependence on concentration is both nonlinear and does not exhibit a power-law relationship. The lack of a linear relationship means that for higher concentrations the filter lifetime may be longer than naively expected. Finally, we observe that the lifetime of the filter falls with with feed concentration but this effect may be mitigated by reducing the flow rate (Fig. 7c).

In Fig. 8 we show iso-lifetime curves: if the concentration of contaminant in the feed were to change, then these curves show how the flow rate must change to achieve a particular lifetime (Fig. 8a) and the improvements that are garnered by increasing the filter size (Fig. 8b). Since in many situations the required flow rate is a fixed quantity one might use this information to determine how many filters should 
$\mathcal{Q}_{\mathrm{i}}$

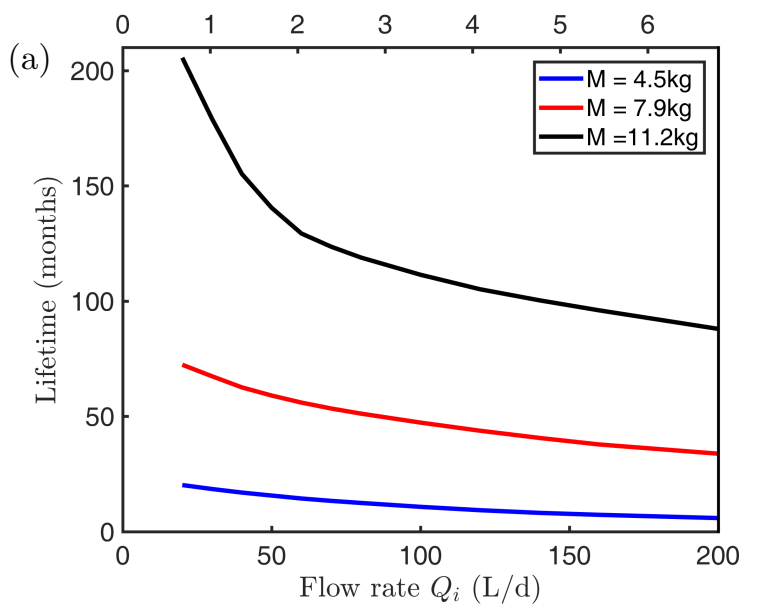

$\mathcal{C}_{\mathrm{f}}$

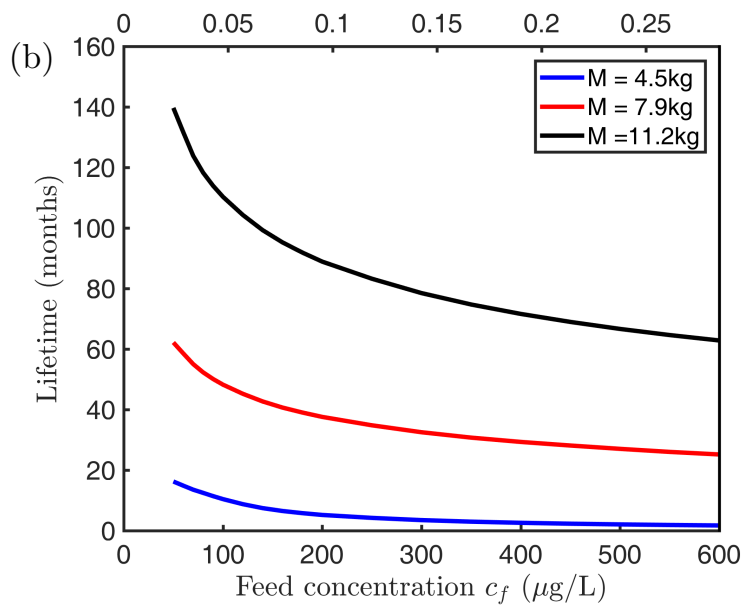

$\mathcal{Q}_{\mathrm{i}}$

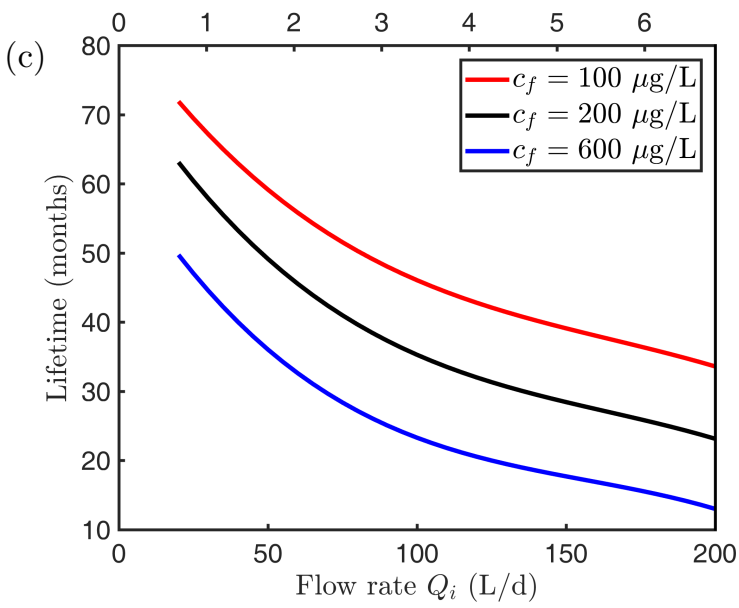

Figure 7: Variation of predicted lifetime of the filter for changes in (a) feed flow rate at different adsorbent dosage and fixed feed concentration, (b) feed concentration at different adsorbent dosage and fixed feed flow rate, and (c) feed flow rate with varying feed concentration at fixed adsorbent dosage. The values of the parameters considered here correspond to the field-scale filter, as listed in Table 3. On the lower $x$ axis we show the dependence on real physical variables for $c_{\mathrm{f}}=100 \mu \mathrm{g} / \mathrm{L}, Q_{0}=100 \mathrm{~L} / \mathrm{d}, R=0.1 \mathrm{~m}$ and $L=0.35 \mathrm{~m}$. On the upper $x$ axis we show the dependence on the core dimensionless parameters defined in (37). The values of $\mathcal{M}$ corresponding to the legends in (a) and (b) are 45, 79 and 112. The values of $\mathcal{C}$ corresponding to the legend in (c) are $0.047,0.095$ and 0.285 . While the dimensional scales apply to a single filtration device, the dimensionless scales enable predictions to be made for the filter lifetime for a range of field-scale filters. 
$\mathcal{Q}_{\mathrm{i}}$

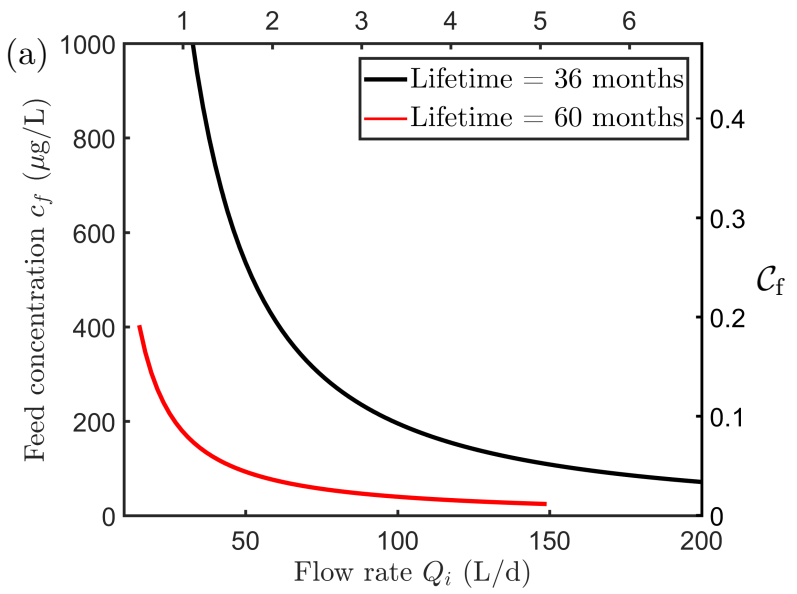

$\mathcal{Q}_{\text {i }}$

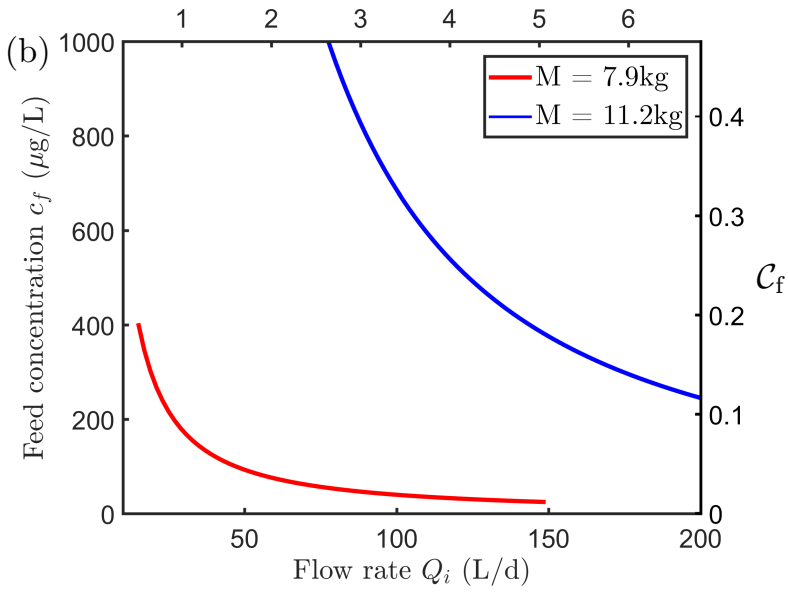

Figure 8: Iso-lifetime curves, along which the lifetime of the filter is constant, where filter lifetime is designated as the time when the output concentration exceeds $10 \mu \mathrm{g} / \mathrm{L}$. In (a) we present two different lifetimes, 36 and 60 months for a filter with $M=7.9 \mathrm{~kg}(\mathcal{M}=79)$. In (b) we present two different filter masses, $M=7.9 \mathrm{~kg}(\mathcal{M}=79)$ and $M=11.2 \mathrm{~kg}$ $(\mathcal{M}=112)$ corresponding to a lifetime of 60 months. The other values of the parameters considered here correspond to the field-scale filter, as listed in Table 3.

be run in parallel if they could only to be exchanged at set times due to restrictions on the frequency of maintenance visits.

For the utility of the filter from an end-user perspective, it is important to have a set of designperformance curves, which can provide the information on the desired lifetime as a function of the feed concentration, amount of adsorbent and the throughput. The information embedded within the graphs presented in Figs. 7 and 8 predicts the lifetime of a given filter, for example for a family home, and offer a method for determining how the filter should be modified to obtain the required lifetime under different operating conditions, such as upscaling to a school or community. This information can thus be used as a tool for the design of the filter for $A s$, or indeed other adsorption-based, filtration, which can be modified according to a specific requirement. 


\section{Conclusions}

No water-purification route is complete without the knowledge about its long-term behaviour, the safe operating lifetime, and the routes for controlling the characteristics of purified water. In this work we have shown how a model based on Langmuir kinetics can be used for the prediction of a fixed-bed adsorption column. Our model incorporates fluid transport, contaminant transport and adsorption on both the interparticle and intra-particle scales. The fluid transport was modelled by the Darcy-Brinkman equation, and reaction-advection-diffusion equations to describe the inter-particle and intra-particle regions, to ultimately understand the adsorption behaviour of the contaminant in the filter. The intra-particle kinetic model was used for the first time for the prediction of filter lifetime.

Through non-dimensionalisation and proper identification of the system parameters, the set of partial differential equations was solved following finite-element schemes with specialised software. The simulation results were compared with simple experimental studies conducted for a single-bed column of raw laterite or treated laterite and values of the effective diffusion coefficients were determined. The resulting model was then free of any fitting parameters and was validated for a dual-bed column of raw and treated laterite. This model was further tested for different operating conditions (feed flow rate, contaminant concentration and filter height) and was proven to be effective in prediction of the system performance in those conditions.

The model was used to analyse the $A s$ transport and adsorption behaviour within the filter media, both on the inter- and intra-particle scales. This gave insight into the inherent multiscale behaviour of the filter that could not be extracted through the experimental measurement of bulk properties, which manifested itself through non-power law dependencies for the filter lifetime and multiple timescale behaviour.

Three core dimensionless system parameters were identified, which characterise the key variables in the As filter challenge: the required flow rate, the contaminant concentration, and the mass of laterite soil used. We showed how the model could predict the dependence of the filter lifetime on these core parameters. The filter lifetime vary significantly with these parameters, ranging from less than 10 months for small filters $(4.5 \mathrm{~kg})$ processing at high flow rates $(200 \mathrm{~L} / \mathrm{d})$ or heavily contaminated $(\sim 500 \mu \mathrm{g} / \mathrm{L})$ water, and over 100 months for larger filters $(11.2 \mathrm{~kg})$ processing water at lower flow rates $(<50 \mathrm{~L} / \mathrm{d})$ or lower contaminant concentrations $(100 \mu \mathrm{g} / \mathrm{L})$. We extracted non-power-law trends that would be difficult to predict even qualitatively in the absence of the model and would take many years to obtain experimentally. We showed how the model analysis could be presented in the form of iso-lifetime curves, which allow for the simple prediction of filter design to achieve a given lifetime, and how a filter should be modified if, for example, the contaminant concentration or the required flow rate were to change.

While the model results were used to provide a set of characteristic curves that act as an assistive tool for the design of $A s$-removal filters, the results readily generalise for the removal of other contaminants via adsorption-based filters. The core result of this work is the systematic reduction of a multiscale model to a set of characteristic curves which bypasses the need for infeasibly long experiments to provide quick and simple predictions for adsorption-based filters. We hope that this work may be adopted to assist in the future deployment and management of these arsenic filters. 
${ }_{398}$ The collaborative work between the two institutions was initiated by the Global Challenges Research 399 Fund. IMG gratefully acknowledges support from the Royal Society through a University Research 400 Fellowship. The authors acknowledge helpful discussions with Lucy Auton, Graham Baird and Doireann 401 O'Kiely. RM gratefully acknowledges support from the the Royal Society through a Challenge Grant. SM gratefully acknowledges support from the Royal Society and EPSRC. The group from the Indian 403 Institute of Technology Kharagpur gratefully acknowledges the inter-disciplinary collaborative initiative 404 taken up by the Mathematical Institute at the University of Oxford. 
Nomenclature

407

$408 \quad(r, z) \quad$ cylindrical coordinates, $\mathrm{m}$

${ }_{409} \mathcal{A} \quad 3 k_{\mathrm{f}}(1-\phi) L / v_{\mathrm{i}} a_{\mathrm{p}}$

${ }_{410} \mathcal{B} \quad \rho_{\mathrm{p} \_} \mathrm{p}^{2} q_{\mathrm{m}} / c_{\mathrm{f}} L^{2}$

${ }_{411} \mathcal{C}_{\mathrm{f}} \quad$ dimensionless measure of contaminant concentration

${ }_{412} \mathcal{M}$ dimensionless measure of filter mass

${ }_{413} \mathcal{Q}_{\mathrm{i}} \quad$ dimensionless measure of volumetric flow rate

${ }_{414} \mathcal{R}^{2} \quad$ linear regression coefficient

${ }_{415} A$ cross-sectional area of filter, $\mathrm{m}^{2}$

${ }_{416} \quad a_{\mathrm{p}} \quad$ particle radius, $\mathrm{m}$

${ }_{417} \quad c \quad$ contaminant concentration, $\mathrm{kgm}^{-3}$

${ }_{418} \quad c_{0} \quad$ initial arsenic concentration in experiment, $\mathrm{kgm}^{-3}$

${ }_{419} \quad c_{\mathrm{e}} \quad$ initial arsenic concentration in experiment, $\mathrm{kgm}^{-3}$

${ }_{420} \quad c_{\mathrm{f}} \quad$ contaminant feed concentration, $\mathrm{kgm}^{-3}$

${ }_{421} c_{\mathrm{p}} \quad$ contaminant concentration at the particle surface, $\mathrm{kgm}^{-3}$

${ }_{422} D_{\mathrm{l}} \quad$ effective diffusion coefficient of contaminant in porous bed, $\mathrm{m}^{2} \mathrm{~s}^{-1}$

${ }_{423} D_{\mathrm{p}} \quad$ diffusion coefficient of contaminant inside particle, $\mathrm{m}^{2} \mathrm{~s}^{-1}$

${ }_{424}$ Da Darcy number

${ }_{425} \quad K_{0} \quad$ adsorption equilibrium constant, $\mathrm{m}^{3} \mathrm{~kg}^{-1}$

${ }_{426} \quad$ adsorption rate, $\mathrm{m}^{3} \mathrm{~kg}^{-1} \mathrm{~s}^{-1}$

${ }_{427} \quad k_{2} \quad$ desorption rate, $\mathrm{s}^{-1}$

${ }_{428} k_{\mathrm{f}} \quad$ mass-transfer coefficient, $\mathrm{ms}^{-1}$

${ }_{429} L \quad$ filter length, m

${ }_{430} M \quad$ mass of adsorbent, $\mathrm{kg}$

${ }_{431} \quad p \quad$ fluid pressure, $\mathrm{Pa}$ 


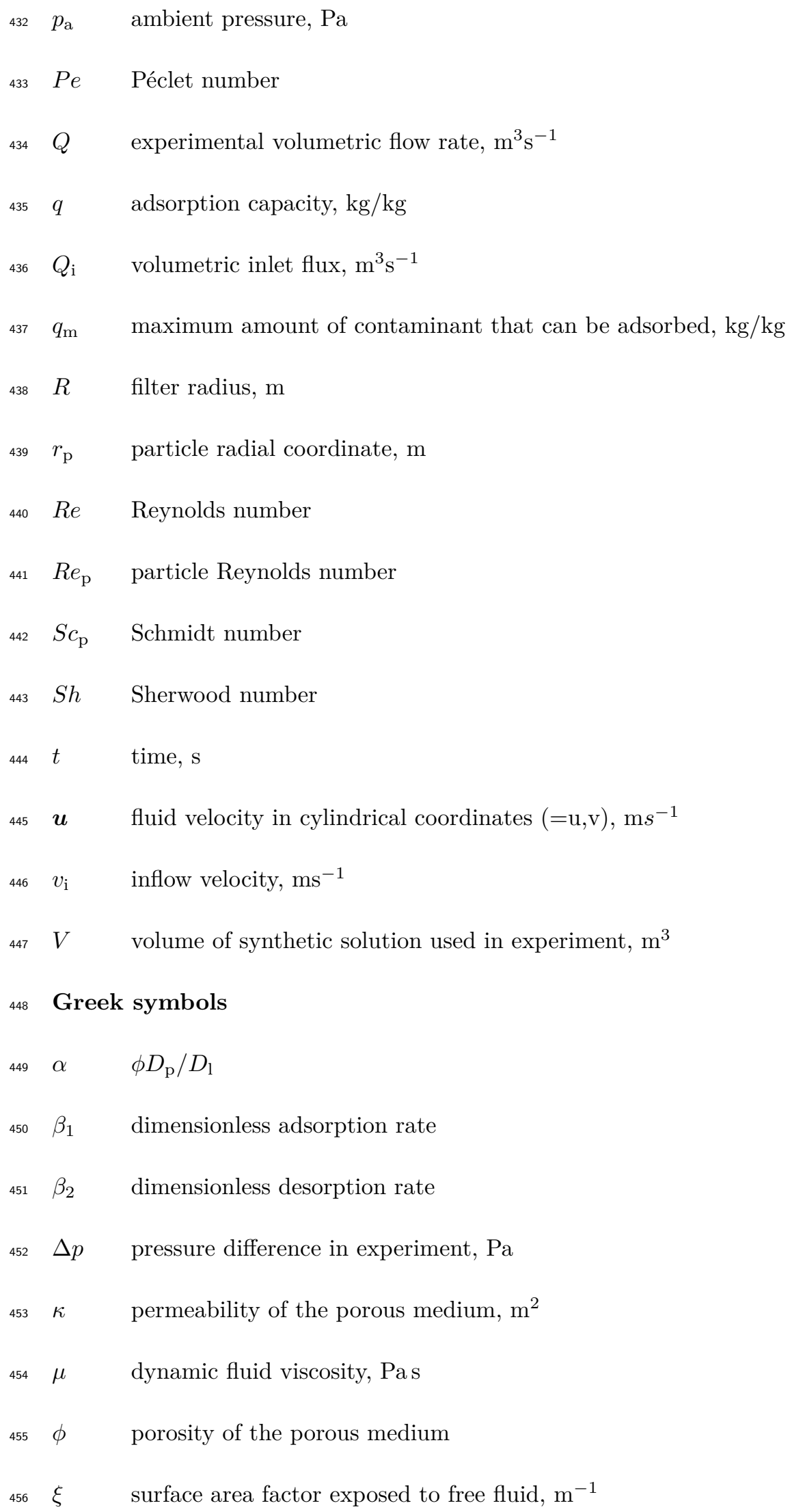




\section{References}

[1] H. N. Tran, S.-J. You, A. Hosseini-Bandegharaei, and H.-P. Chao. Mistakes and inconsistencies regarding adsorption of contaminants from aqueous solutions: a critical review. Water Research, 120:88-116, 2017.

[2] M. Leist, R. J. Casey, and D. Caridi. The management of arsenic wastes: problems and prospects. J. Haz. Mat., 76(1):125-138, 2000.

[3] J. C. Ng, J. Wang, and A. Shraim. A global health problem caused by arsenic from natural sources. Chemosphere, 52(9):1353-1359, 2003.

[4] I. A. Katsoyiannis and A. I. Zouboulis. Removal of arsenic from contaminated water sources by sorption onto iron-oxide-coated polymeric materials. Water research, 36(20):5141-5155, 2002.

[5] WHO reports. Arsenic proposed drinking water regulation: A science advisory board review of certain elements of the proposal. US EPA, EPA-SAB-DWC 1-001, 2000, https://nepis.epa.gov/exe/zypurl.cgi?dockey=p100fl7r.txt.

[6] A. Maiti, S. DasGupta, J. K. Basu, and S. De. Batch and column study: adsorption of arsenate using untreated laterite as adsorbent. Industrial \& Engineering Chemistry Research, 47(5):1620$1629,2008$.

[7] A. Maiti, H. Sharma, J. Kumar Basu, and S. De. Modeling of arsenic adsorption kinetics of synthetic and contaminated groundwater on natural laterite. J. Haz. Mat., 172(2-3):928-934, 2009.

[8] A. Maiti, J. K. Basu, and S. De. Experimental and kinetic modeling of as (v) and as (iii) adsorption on treated laterite using synthetic and contaminated groundwater: Effects of phosphate, silicate and carbonate ions. Chem. Eng. J., 191:1-12, 2012.

[9] S. K. Maji, A. Pal, T. Pal, and A. Adak. Sorption kinetics of arsenic on laterite soil in aqueous medium. J. Environ. Sci. and Health, Part A, 42(7):989-996, 2007.

[10] G. S. Bohart and E. Q. Adams. Some aspects of the behavior of charcoal with respect to chlorine. J. American Chem. Soc., 42(3):523-544, 1920.

[11] A. Wolborska. Adsorption on activated carbon of p-nitrophenol from aqueous solution. Water Research, 23(1):85-91, 1989.

[12] S. Mondal, A. Roy, R. Mukherjee, M. Mondal, S. Karmakar, S. Chatterjee, M. Mukherjee, S. Bhattacharjee, and S. De. A socio-economic study along with impact assessment for laterite based technology demonstration for arsenic mitigation. Science of The Total Environment, 583:142-152, 2017.

[13] K. Tsiberkin. Effect of inertial terms on fluid-porous medium flow coupling. Transport in Porous Media, 121(1):109-120, 2018. 
[14] J. M. Coulson, J. F. Richardson, J. R. Backhurst, and J. H. Harker. Chemical Engineering Vol 2. Pergamon Press, Oxford, 1968.

[15] Y. Liu and L. Shen. From langmuir kinetics to first-and second-order rate equations for adsorption. Langmuir, 24(20):11625-11630, 2008.

[16] J. M. Coulson, J. Francis Richardson, J. R. Backhurst, and J. H. Harker. Chemical Engineering Vol 1. Pergamon Press, Oxford, 1968.

[17] F. H. Garner and R. B. Keey. Mass-transfer from single solid spheres-I: Transfer at low reynolds numbers. Chem. Eng. Sci., 9(2-3):119-129, 1958.

[18] B. S. Persons. Laterite: Genesis, Location, Use. Springer Science \& Business Media, 2012.

[19] M. Lunt. Introduction to statistical modelling: linear regression. Rheumatology, 54(7):1137-1140, 2013.

[20] S. Gupta and B. V. Babu. Modeling, simulation, and experimental validation for continuous $\mathrm{Cr}$ (VI) removal from aqueous solutions using sawdust as an adsorbent. Bioresource Technology, 100(23):5633-5640, 2009.

[21] WHO Chronicle. Guidelines for drinking-water quality, fourth edition. 38(4):104-8, 2011. 


\section{Supplementary Information}

\section{Kinetic experiments}

The kinetic experiments were carried out by measuring the concentration of the solution at different instants of time. For this, nine conical flasks each having $100 \mathrm{ml}$ of feed (aqueous solution of arsenic of known concentration) were taken and known weight of adsorbent was added into that. These flasks were then maintained under continuous shaking (speed $150 \mathrm{rpm}$, temperature $25^{\circ} \mathrm{C}$ ). Each flask was removed at different time points and samples were analyzed for arsenic concentration. This concentration was used to calculate the adsorption capacity $(q)$ at that instant of time. The $q$ values were thus calculated at nine time points and used to calculate the kinetic constants using Eq. (31). A plot of $c / c_{0}$ (where $c_{0}$ is the initial concentration) with time for RL and TL is shown in Figure S1.

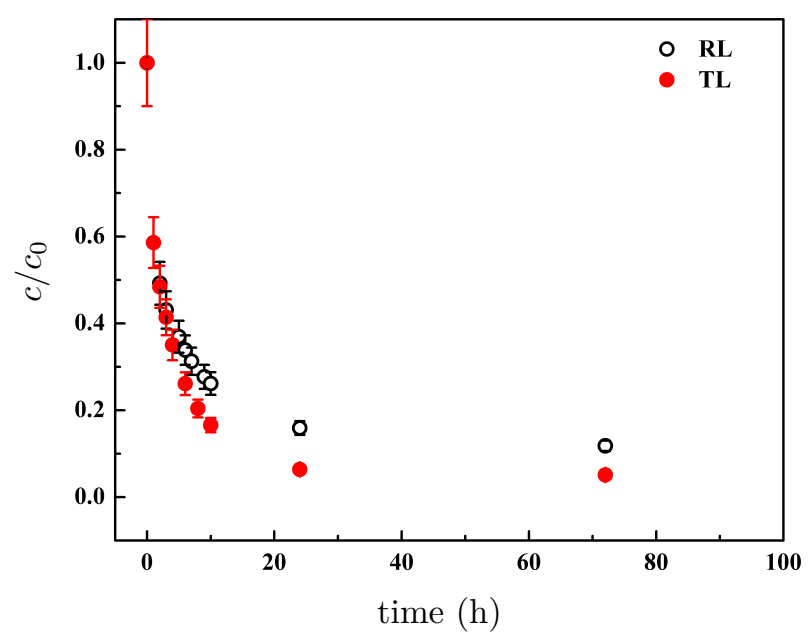

Figure S1: Plot of $c / c_{0}$ with time for RL and TL during kinetic study. 